Seta, J. J., Seta, C. E., \& McElroy, T. (2002). Strategies for educing the stress of negative life experiences: An averaging/summation analysis. Personality and Social Psychology Bulletin, 28: 1574-1585. Published by SAGE (ISSN: 0146-1672).

\title{
Strategies for Reducing the Stress of Negative Life Experiences: An Averaging/Summation Analysis
}

\author{
John J. Seta, Catherine E. Seta, and Todd McElroy
}

\begin{abstract}
Experiments 1 through 4 investigated how different orientations to stimulus events influenced whether the addition of a mildly negative stressor to a highly negative one did or did not decrease stress. In Experiment 1, reductions in stress levels were obtained when perceivers concentrated on the negative implications of each stressor but not when they concentrated on the implications associated with the entire situation. In Experiments 2 and 3, reductions in stress were obtained when participants were asked to think about a mildly negative event in addition to a highly negative one, but this effect was reduced when participants overtly appraised each stressor before reporting their overall stress reaction. In Experiment 4, stress reductions were obtained when participants were induced to think about either two or four of the negative implications associated with a single stressor but not when they thought about six negative implications.
\end{abstract}


Early conceptions of stress were concerned with physiological responses to environmental stressors and not on how individuals appraised the meaning of these events (e.g., Seyle, 1956). It was not until the work of Lazarus and his associates (e.g., Lazarus, 1966; Lazarus, Delongis, Folkman, \& Gruen, 1985; Lazarus \& Folkman, 1984 ) that the central role of appraisal in the production of stress reactions was recognized.Lazarus et al.(1985), for example, argued that "a full understanding of stress reactions involves how a person and environmental antecedent variables interact to produce divergent appraisals that reflect whether the encounter is irrelevant, benign, or stressful, and if stressful, what might be done to cope" (pp.776-777).Although the importance of the appraisal process is now well recognized, important aspects of this process, such as how individuals integrate components of life events, have been largely neglected. This article is concerned with this topic.

Research by Seta, Seta, and colleagues has explored factors that influence the way individuals integrate life stressors (e.g., C.E.Seta \& Seta, 1992, 1996; J.J.Seta, Crisson, Seta, \& Wang, 1989; J.J.Seta, Seta, \& Wang, 1991; J.J.Seta, Wang, Crisson,\&Seta, 1989).Specifically, they were concerned with whether experiencing a highly negative event coupled with a less negative one would produce increasingly negative reactions. Or could experiencing a less negative event instead attenuate the effect of the highly negative one? Their research demonstrated that both effects are possible and developed an averaging/ summation (AS) model of stress integration that describes the integration strategies leading to both outcomes.

According to the AS model (e.g., C. E. Seta \& Seta, 1992, 1996; J.J.Seta et al., 1989), people are sensitive both to central tendencies and to the frequency and overall intensity of life events.A veraging is conceptualized as a process that establishes a central tendency among (between) stimuli.In an audience setting, each audience member can be a distinct source of consequences to performers in, for example, influencing their public and private self-image.Because of this, the overall consequences that can be administered by each audience member are typically a critical concern for performers. Therefore, in addition to seeking a central tendency between (among) the implications associated with each audience member (expressed, e.g., as the average status of the audience), the implications associated with each audience member also can be summed and expressed as the total impact of all audience members. 
Because people are sensitive to both of these dimensions, the addition of an evaluative event can lower or raise the level of stress associated with the situation. For example, the addition of a relatively low-status audience member to an audience of two high-status members can lower impact and stress levels because it lowers the overall status of the audience.In this situation, overall status is an average or central tendency impression of group members. Thus, the low-status member reduces the impact and stress associated with the audience (C.E.Seta \& Seta, 1996).(See the General Discussion for other ways in which the addition of a low-level stimulus can reduce impact from our model.) The addition of a relatively low-status member, however, can also raise impact and stress in that the audience member can raise the total amount of consequences that the audience can deliver and thereby increases the performer's stress level.

In general, the model states that a person's overall response represents the outcome of how he or she integrates events. Two integration strategies are specified:

One strategy is a simple summation process in which the events are integrated in an additive manner and the other is a strategy of event averaging that results in the formulation of a central tendency value representing the average level of the events present in the context. The model specifies that persons utilize both integration strategies, although each strategy may be differentially weighted in different contexts. The resulting response experienced by a person is an outcome of his or her sensitivity to these processes.

The AS model can be expressed more precisely as follows:

$R=\frac{V_{1}\left[\frac{W_{0} S_{0}+W_{1} S_{1}+W_{2} S_{2}}{W_{0}+W_{1}+W_{2}+\mathrm{K}}+\mathrm{K}\right]+V_{2}\left[W_{0} S_{0}+W_{1} S_{1}+W_{2} S_{2}+\mathrm{K}\right]^{i<1}}{V_{1}+V_{2}}$

The first bracketed section contains an averaging rule, the second bracket contains a summation rule. $R$ refers to the individual's overall response (e.g., stress level); $S$ refers to stimulus value, previously described as scale value (e.g., J. J. Seta et al., 1989); S0 represents the initial state of the individual as he or she enters the context as well as the individual's initial expectation concerning 
the type of information that will be contained in the context; S1, S2 refers to the defining features of each stimulus such as its properties and implications; $W$ refers to the weight or influence of the stimulus that is determined by factors such as importance or attention; $V$ refers to the strength or influence of these two integration rules; $V 1$ corresponds to the strength of averaging; and $V 2$ refers to the strength of summation.1

Essentially, this formula expresses a method of integrating various components of a setting and includes individuals' sensitivities to both the average (or central tendency) impact of the various components (first bracketed section) and the summative (or accumulated) impact of every individual component (second bracketed section). Each stimulus within the context (S) contributes to the determination of a central tendency (the average) and the summative impact of the components, and these stimuli combine to produce an overall response to the setting $(\mathrm{R})$.Each stimulus has a value along some dimension of judgment. These values may be along dimensions such as the magnitude of negativity, positivity, status level, confidence, or consequences.In addition, the summation procedure is raised to a power (t) with an exponent less than 1 to reflect the commonly found marginally decreasing utility function for redundant features of a setting.

The vast majority of research (e.g., C. E. Seta \& Seta, 1992, 1996; J.J.Seta et al., 1989, 1991) has focused on the model's prediction concerning stimulus similarity.Specifically, when two stimuli have very similar stimulus values (e.g., two high-status audience members), the influence of the two events in combination will be greater than either event in isolation.For example, adding an additional high-status person to an audience composed of one high-status person should result in an increment in anxiety. When two stimuli are relatively discrepant, however, the influence of the average of the two stimuli can be significantly less than either event in isolation.So, for example, when we add a low-status audience member to an audience of a high-status member, the addition of the low-status member can significantly reduce the overall status of the group (e.g., the average amount of consequences associated with each audience member) while only marginally increasing the total amount of consequences that the audience can administer.Therefore, in this context, the addition of a low-status audience member will lower the audience's impact and the individual's stress level.Research has supported this prediction.In one study, reductions in anxiety were found when high 
school students were added to an audience of faculty but not when undergraduates were added to an audience of graduate students (J.J.Seta et al., 1989).Because the difference in the stimulus values of graduate and undergraduate students is relatively small, their average can only produce small reductions in impact.Consequently , the relative impact of summation is magnified.Similar findings were obtained when high school students were added to an audience of high school students or when faculty were added to an audience of faculty (e.g., J. J. Seta et al., 1989). There are boundaries, however, to this effect in that reductions in impact or stress cannot occur if perceivers do not attend to the implications of the lowimpactful stimulus. This situation can occur when a highly negative stimulus is so intense (in a relative or absolute sense) that perceivers do not attend to the addition of a mildly negative stressor. This result was seen in a study by Seta and Seta (1996) in which high- and lowsocially anxious participants expected to interact with other persons. Because high-socially anxious people have been shown to concentrate an especially large amount of their attention on high-status audience members, the addition of a low-status member to a discussion group consisting of a high-status member was expected to reduce the stress level of low- but not that of highsocially anxious participants. Results confirmed these predictions. It took the attention-grabbing properties of three low-status members to significantly reduce the stress levels of high-socially anxious participants.

Results that confirm predictions of the model have been obtained across several different contexts and with several different dependent variables.In studies that tested the AS model using within-subjects designs, individuals were asked to report the amount of anxiety they would feel while performing in front of various homogeneous and heterogeneous audiences (e.g., J. J. Seta et al., 1989; C.E.Seta \& Seta, 1996) or while imagining themselves confronted with a variety of negative life events (e.g., J. J. Seta et al., 1991). Supportive results using negative life events also have been obtained in nonsimulation studies using a between-subjects design (e.g., J. J. Seta et al., 1991). This study employed a manipulation commonly used to induce affective states (Schwarz \& Clore, 1983), one in which participants were asked to experience the negative event(s) by writing about the negative implications associated with each event before reporting their feelings of stress. Results revealed both stress-averaging and summation effects. 
In addition to verbal reports, supportive results have been found using behavioral and physiological measures. J.J.Seta et al.(1989) found behavioral support for the averaging/summation model when participants performed a paired-associates task in front of either a homogeneous or heterogeneous audience.In addition, C.E.

Seta and Seta (1992) found physiological evidence in an audience setting. In this study, the addition of one high school student to an audience of one faculty member lowered mean arterial blood pressure, whereas the addition of the same student to an audience of one high school student raised blood pressure.

\section{EXPERIMENT 1}

The AS model describes how the same stimulus event can lead to both reductions and increments in impact. The present series of studies explored how the use of different modes of responding to the configuration of stressful life events influenced participants' level of stress.When faced with multiple stressors, individuals have several options for considering the implications of these events. They may think about and react to the implications of each stressor independently and then formulate an integrated response.In this context, considering the separate implications of the high and low stressors provides the opportunity for participants to see the distinctiveness of the stressing events, thus providing the opportunity for an integration of these events in terms of averages or central tendencies.As seen in the previous research on reactions to heterogeneous audiences (see previous discussion and J.J.Seta et al., 1989), it is necessary for there to be a significant difference between the stimulus values of relevant stimuli to observe a significant impact of averaging processes. Whenthe stimulus values of stimulus events are relatively similar, averaging would produce small reductions in impact; consequently, the relative impact of summative processes is magnified in participants' responses. In the present study, it was expected that inducing participants to consider the separate implications of a high- and lowlevel stressor would allow the stimulus value differences that exist between these stressors to emerge, resulting in a stress-averaging effect.

On the other hand, rather than thinking about the separate implications of stressors, individuals can consider the implications of the entire situation.By directing participants to consider the implications of an entire situation, containing both high and low stressors, participants 
may be more likely to think of "all the consequences" of the stimuli, so that additional consequences

are considered "on top of" other events.Indeed, communal definitions of the term total include the term "entire": "total: constituting or comprising the whole of something; entire"; "sum; aggregate" (The Random House College Dictionary, 1984, p.1388).In this case, we would expect that the addition of a stressor would either not lower stress or that its stress-reducing influence would be relatively weak.

To test this prediction, Experiment 1 included three conditions. In the separate condition, participants were directed to list four negative implications of a highly negative event and four negative implications of a mildly negative one.In the total condition, participants were directed to list eight negative implications that were associated with the entire situation.T o provide a basis for comparing the responses of participants in the separate and total conditions, we included a highly negative event control condition, one in which participants listed four negative implications of a highly negative event.Support for our hypotheses would be found if participants' reports of stress are lower in the separate condition than in either the one highly negative control condition or in the total condition.

\section{Method}

Participants and design.Participants included 127 female students from introductory psychology classes who took part in partial fulfillment of a course requirement. Experimental sessions were conducted in groups containing an average of about 10 individuals. Condi tions were randomized within each group so that each session contained an approximately equal number of experimental conditions.We used a between-participants design that included three stress conditions: one highstressor condition and two conditions in which a high and low stressor was used (total and separate implication).

Procedure.Participants were told after entering the experimental room that the experimenter was interested in their reaction to several different issues. In the one highly negative condition, participants were asked to write four negative implications about financial problems. In the separate condition, they were asked to list four negative implications of financial problems and then four negative implications of a lesser stressor- 
home maintenance.In the total condition, participants were asked to list eight negative implications of the entire situation (financial problems and home maintenance). These stressors were taken from an earlier normative study conducted on participants from the same student population as used in the present study with the stipulation that participants could not be in both studies. Norms determined that home maintenance was a lower level stressor relative to financial problems.Order was counterbalanced in both the separate and total conditions. After providing the above information, participants were asked how stressed or unpleasant they felt this situation would make them feel on a 101-point scale where 0 represented not at all and 100 represented extreme stress or unpleasant.In this and in all subsequent experiments, all participants were provided with information on how to contact the research adviser and clinical services in the community if they felt in need of discussing their reactions more fully.Participants were thanked and debriefed.

\section{Results and Discussion}

We performed an ANOVA on these three between conditions: one highly negative, separate, and total.The ANOVA revealed a significant stress configuration condition main effect.2 Participants showed the effects of averaging in the separate condition. The stress scores in the separate condition $(M=60.97, S D=24.3)$ were significantly lower than in the one highly negative condition $(M=77.55, S D=15.32), F(1,124)=14.65, p<.001$. This was not the case in the total condition. The stress scores in this condition $(M=76, S D=18.85)$ did not differ from those in the one highly negative condition, $F<.2$. Furthermore, the stress scores in this condition were higher than those in the separate condition, $F(1,124)=12.17, p<.01 .3$ When participants were asked to concentrate on the negative implications of both stressors in the total condition, they appeared to concentrate on the negative implications of all of the events and each stressor was regarded as a part of the whole.This was, however, not the case in the separate condition.In this condition, participants' attention was drawn to distinctions between the events. This precondition allowed for the integration of the events in terms of the average amount of stress present in the context, and participants reported lower stress levels in this condition than in the one highly negative condition. Thus, this study demonstrated that the likelihood of obtaining stress-averaging effects is influenced by participants' orientations to stressing situations. 
Although the participants in Experiment 1 did not experience the negative life events, they did write about the negative consequences that were associated with these events.By so doing, they put themselves in contact with the negative consequences of these events.

Although seemingly artificial, this type of situation is not restricted to a laboratory setting, as the world events of terrorism have unfortunately shown us.In fact, people are often reminded of negative events (theirs and others) and they often ruminate about these events (e.g., Martin \& Tesser, 1996).By asking our participants to think about the negative implications of events, we are, to an extent, asking them to ruminate (e.g., Lyubomirsky\&Nolen-Hoeksema, 1995; Martin\&Tesser, 1996; Strack, Blaney, Ganellen, \& Coyne, 1985).

Therefore, to the extent that thinking about the negative implications of an event is similar to rumination, the results of Experiment 1 suggest that the potential stressing effects of rumination may depend on the specific integration strategy that a person employs.

\section{EXPERIMENT 2}

Experiment 1 demonstrated that orienting conditions expected to weight averaging versus summative integration strategies resulted in different responses to identical configurations of stressing life. Stress-averaging effects were seen only when participants were oriented to consider the separate implications of the high and low stressors. Orienting participants to consider the implications of the "entire situation" appeared to draw attention away from the distinctions between the stimulus components and emphasized an integration strategy weighting summative versus averaging processes.

In Experiment 2, we further explored the influences of manipulating participants' orientations to identical stress-situation configurations and investigated how the timing of when participants were asked to formulate a response to stressful stimuli influenced the likelihood of engaging in stress-averaging processes. In one condition (final response), participants were first asked to name and think about each stressful event and then they were asked the level of stress that they experienced from thinking about all of the events. This is the basic method that we used in prior studies (e.g., J. J. Seta et al., 1991) as well as in Experiment 1.In the second condition (interpolated response), participants also were asked to name and think about each event. However, before being asked the level of stress they experienced from thinking 
about all of the events, they were asked to report the level of stress that they experienced from thinking about each event, separately. The overt expression of stress should have a strong influence on the tendency to observe stress-averaging effects.

Increasing the salience of a person's overt stress response to individual stressing events should impede any process that would result in reductions of stress below the absolute level of the highest expressed response. For example, if Joe told his therapist, bartender, or friend that he felt "very stressed" by an argument with his spouse, it would be unlikely for him to then say that he felt a lesser amount of stress if this argument occured in the context of another stressor, even one of lower magnitude. Indeed, the averaging effect is counterintuitive, as discussions with our students and colleagues have revealed to us. Therefore, any orientation to stressing situations that makes salient a person's overt appraisal of his or her stress appraisal to individual stressors may have the consequence of impeding the verbal expression of stress-averaging effects. Experiment 2 was designed to assess this prediction.

Our second experiment also was performed to determine if averaging effects would be obtained in a situation in which individuals think and write about events that they themselves experienced directly. To increase the chances that the highly negative event would have important consequences to our participants, they were instructed to think and write about events that were personally traumatic in nature.

If individuals average a mildly negative event and a highly negative event, then their perceptions of stress should be lower than that produced by participants who were only exposed to the highly negative event. Further more, their stress reactions should be higher than that produced by participants who were only exposed to a mildly negative event. Although the addition of a few mildly negative events to a highly negative one should reduce stress levels, the addition of other mildly negative events may raise stress levels above the level produced by the addition of only one or two mildly negative events. This can occur because the further addition of mildly negative events may not significantly alter the average negativity associated with the situation. Nevertheless, these events can increase stress because they add additional negative consequences to a person's life-consequences that deplete the individual's pool of available resources (e.g., Hobfoll, 1988a, 1988b). Therefore, in 
Experiment 2, we also included a condition in which participants were asked to think about a highly negative event and three mildly negative events. Stress levels in this condition may not be below that produced by the highly negative event and the one mildly negative event. In fact, they may be higher.

In sum, Experiment 2 was designed to determine if thoughts about a highly negative event, coupled with thoughts about a mildly negative event, would result in a level of stress that was below that produced by thoughts concerning a highly negative event alone.In addition, we expected to observe stronger stress-averaging effects in the final than in the interpolated response condition.

\section{Method}

Participants and design.Participants included 110 female students from introductory psychology classes, and the sessions were conducted in the manner described in Experiment 1.We used a between-participants design that included two different response formats (final and interpolated) and two levels of mixed stressors (highly negative event plus mildly negative event; highly negative event plus three mildly negative events).In addition, the design included two conditions that did not fit the factorial design.It included a highly negative event condition and a mildly negative event condition that were used as reference points for accessing the impact of the mixed stressor combinations.

Procedure. Participants in the highly negative event condition read that they should take a moment to think about a traumatic event that happened to them. Then they were asked to write the nature of the event in the space provided. After doing so, they turned the page in the booklet and read a question that asked them to indicate how stressed or unpleasant thinking about the traumatic event made them feel, where 0 represented no stress and 100 represented extreme stress.

Participants in the mildly negative event condition and those in the mixed stressor conditions were given identical instructions with the exception that they were asked to recall different types of events. In the mildly negative condition, they were asked to name and think about a mildly negative event that happened to them; in the mixed stressor conditions, they were asked to name and think about one traumatic event and one mildly negative event or one traumatic event and three mildly negative events. Order of events was counterbalanced. Participants in the interpolated response condition 
were given additional instructions. Before being asked how stressed or unpleasant thinking about the events made them feel, they were asked to report the level of stress that they experienced from thinking about each event. Therefore, in addition to thinking about and naming each event, these participants also rated how stressed or unpleasant thinking about each event made them feel, where 0 represented no stress and 100 represented extreme stress.

\section{Results and Discussion}

Participants wrote about a variety of highly negative and mildly negative events. They included the death of a family member or close friend, abuse, and punishment. Examples of mildly negative events included events such as lying, minor fights, and performing poorly on an exam or quiz.4

Because the design was not factorially balanced, we performed a one-way ANOVA on the six experimental conditions. The means for each condition are contained in Table 1.This analysis was performed to provide an appropriate error term for planned contrasts between conditions. The ANOVA revealed a significant conditions effect, $F(5,104)=4.39, p<.001 .5$ To determine if we obtained an averaging effect, we compared stress levels in the highly negative event condition to those in the final and interpolated response conditions. Specifically, we compared the highly negative event condition to the highly negative event plus one mildly negative event. Stress levels in the one high and one mild condition were significantly lower than the level associated with the highly negative event condition alone. In addition, although the stress level associated with the one high event plus three mild condition was somewhat higher than the level associated with the one high and one mild condition, this difference was not significant, $F<1$. The combined stress levels of these two conditions, however, were significantly lower than the level associated with the one high condition, $F(1,104)=10.53, p<.01$, and higher than the level associated with the one mild condition, $F(1,104)=11.58, p<.01$. These results support predictions that the addition of one or a few mildly negative events to a highly negative event context would lower stress levels below that produced by the highly negative event itself. 
TABLE 1: Mean Stress Ratings for Each of Our Six Experimental Conditions

\begin{tabular}{lcc}
\hline Condition & $\mathrm{M}$ & $\mathrm{SD}$ \\
\hline $\begin{array}{l}\text { Highly negative event } \\
\begin{array}{l}\text { Mildly negative event } \\
\text { Final evaluation—highly negative event }\end{array}\end{array}$ & 73.72 & 21.75 \\
$\begin{array}{l}\text { and one mildly negative event } \\
\text { Final evaluation—highly negative event } \\
\text { and three mildly negative events }\end{array}$ & 47.47 & 27.95 \\
$\begin{array}{l}\text { Interpolated evaluations—highly negative } \\
\text { event and one mildly negative event }\end{array}$ & 54.74 & 27.21 \\
$\begin{array}{l}\text { Interpolated evaluation—highly negative } \\
\text { event and three mildly negative events }\end{array}$ & 60.29 & 28.58 \\
\hline
\end{tabular}

We also expected to obtain reduced averaging in the interpolated response condition. Although adding a mildly negative event to a highly negative event reduced stress in the interpolated response condition, this effect was not significant, $F(1,104)=2.46, p<.15$. This comparison was significant in the final response condition, $F(1$, $104)=9.42, p<.01$. In addition, the stress levels in the interpolated response condition were higher than those in the final response conditions, $F(1,104)=4.11, p<.05$. We also computed stress differences in each of the response format conditions (final and interpolated) relative to the highly negative condition. Then, we compared these differences. This contrast was significant, $F(1,104)=4.08, p<.05$. These findings support our reasoning that asking participants to overtly express their reactions to a stressing event decreases the likelihood of obtaining stress averaging.

These results also provide evidence that the addition of a mildly negative event to a highly negative one can reduce feelings of negativity and stress in a situation in which participants think and write about events that they have experienced directly. In this way, it is similar to previous audience studies that have supported the averaging/ summation model (e.g., C. E. Seta \& Seta, 1992; J. J. Seta et al., 1991).

\section{EXPERIMENT 3}

In Experiment 1, we found that the addition of a relatively mild stressing event to a highly stressing one reduced stress when participants used a final response procedure and first listed the negative implications of each stressor.In Experiment 2, the stress-mitigating 
effect of first considering each stressor, separately, was reduced when participants were induced to use an interpolated response procedure. Thus, although both manipulations appear on the surface to induce the separation of stressors, it is clear that the interpolated response technique has a quite different effect. Indeed, separating the stressing events through requirements to express current levels of experiential stress had the effect of decreasing the likelihood of stress averaging. We expected this effect based on considerations that stressor averaging is, indeed, a counterintuitive effect that we did not expect participants to be able to predict. Thus, self-presentation and consistency factors were expected to mitigate the potentially beneficial effect of stressor averaging, leading individuals to express reduced stress-averaging effects in this condition. Study 3 was designed to further test the effects of interpolated response procedures in a context in which stressor separations also are induced by the implication listing task used in Experiment 1.Thus, we explored whether the interpolated response procedure used in Experiment 2 would still reduce the stress-mitigating effects of a final response procedure when participants also had listed the negative implications of each stressor, as in Experiment 1.We also used different types of stressors in this experiment to increase generalizability and to reduce the possibility that the results of Experiment 2 were due to the specific stressors that were used.

\section{Method}

Participants and design. Participants included 90 female students from introductory psychology classes, and the sessions were conducted in the manner described in Experiment 1.We used a between-participants design that included three conditions: a highly negative event condition (traumatic experience) and two mixed stressor conditions (one traumatic experience plus one mildly negative event). The mixed stressor conditions used either a final or an interpolated response format. Procedure. The general instructions given to participants were similar to those used in Experiments 1 and 2. In the one highly negative condition, participants were asked to write four negative implications about a traumatic experience. Then, they were asked to indicate how stressed (or unpleasant) thinking about this situation made them feel. Participants in the final mixed condition were given identical instructions except they were asked four negative implications of two events-traumatic experience and one mildly negative event. Order 
of stimulus presentation was counterbalanced across participants. Participants in the interpolated-mixed condition were given an additional assignment: Before being asked how stressed thinking about the events made them feel, they were asked to report the level of stress that they experienced from thinking about each event. Therefore, in addition to listing the negative implications of each event, they also rated how stressed (or unpleasant) thinking about each event made them feel, where 0 represented no stress and 100 represented extreme stress. Participants were given contact information, thanked, and debriefed.

\section{Results and Discussion}

We performed a between-participants ANOVA on participants' overall stress reactions in the three experimental conditions. Because the order in which the stressors were presented was not significant, $F<1$, and because it did not interact with our experimental conditions, this factor was collapsed across conditions. The ANOVA revealed a significant condition main effect, $F(2,87)=$ $11.48, p<.001$. The stress scores of participants in the final $(M=73.04, S D=17.1)$ and interpolated mixed $(M=$ 82.37, $S D=12.8$ ) conditions were lower than in the one highly negative condition $(M=89.41, S D=9.1), F(1,87)=$ $22.92, p<.001 ; F(1,87)=4.39, p<.05$, respectively. Furthermore, the stress scores in the final-mixed condition were significantly lower than in the interpolated-mixed condition, $F(1,87)=7.22, p<.01$. These results provide a conceptual replication for the results that were found in the final and interpolated response conditions of Experiment 2.Consequently, it appears that an interpolated response procedure reduced the mitigating influence of adding a relatively low-level stressor under conditions in which participants were asked to list the negative implications of each stressor. These results support the reasoning that requiring participants to overtly express their reactions to a stressing event reduces the likelihood of obtaining stress-averaging effects.

\section{EXPERIMENT 4}

The results of Experiments 1 to 3 demonstrated that by considering the individual contributions of stressors, the addition of a relatively low stressor to a relatively high one can reduce stress reactions. A conceptually related issue is whether thoughts about a few of the negative implications that are associated with a single stressor will 
produce results that are similar to thinking about individual stressors. For example, if individuals think about an entire event as well as a few of the negative implications that are associated with this event, they are, in effect, separating the event into two parts: (a) the event itself and (b) the implications that are a subset of the event.In separating the event in this way, we expected that just as the addition of one or a few mildly negative events can reduce stress, so should thoughts about a few of the negative implications associated with a single stressor.In addition, just as the addition of many mildly negative events can increase stress, so should thoughts about many of the negative implications associated with a single stressor.Experiment 4 was designed to assess the validity of these assumptions.

\section{Method}

Participants and design. Participants included 138 female introductory psychology students, and the general procedures used in the previous study were employed. We used a between-participant design that included four experimental conditions. In the highly negative experience condition, participants were asked to think about a traumatic experience that had occurred to them. In the negative implication conditions, participants were asked to think about a traumatic experience that had occurred to them and either two, four, or six negative implications that were associated with this experience.

Procedure.All participants were presented with a two-page booklet that contained the same general instructions:

"We would like you to take a moment to think about a traumatic event that had very negative implications for you and then to answer the question(s) that follow." Participants in the traumatic only condition (control condition) were provided with a space to indicate that nature of the traumatic event. Parallel to control participants, participants in the experimental conditions also were asked to provide the nature of the traumatic event. In addition, these participants also were asked to write down and then rate either two, four, or six implications of the negative event. The implications were rated individually using a 101-point scale where 0 represented the least negative aspect of the event, 50 represented average, and 100 represented the worst aspect of the event. Participants were then asked, "How stressed or unpleasant does thinking about this traumatic event make you feel?" Ratings were made on a scale in which 0 
represented not at all stressed or unpleasant and 100 represented extremely stressed or unpleasant. Following these procedures, participants were given contact information, debriefed, and thanked.

\section{Results and Discussion}

Participants indicated a variety of highly negative experiences. Some of the most common examples of highly negative experiences that participants expressed were death of a family member or close friend, illness, and car accidents. The implications that participants wrote down concerning the highly negative experience were consistent with the highly negative experience itself. For example, when dealing with the death of a family member, some noted implications were depression, fear of death, and loss of the person's company. We performed an ANOVA on participants' reports of stress. The means for each of the four experimental conditions are contained in Table 2.The ANOVA revealed a significant main effect for condition, $F(3,128)=5.34, p<$ .05. A planned contrast revealed that there was more stress reported in the six than in the four negative implication condition, $F(1,128)=7.07, p<.01$. More important, however, there was less stress reported in the four negative implications condition than in either the highly negative experience condition or in the two negative implications condition, $F(1,128)=11.46, p<.01 ; F(1$, $128)=3.97, p<.05$, respectively. There was also a tendency for participants to report less stress in the two negative implications condition than in the highly negative experience condition, $F(1,128)=2.13, p<.15$. These results support predictions derived from an averaging/ summation model. They suggest that thinking about the negative implications that are associated with a highly negative experience lowers stress levels below that produced by the highly negative experience alone. Another interpretation of the data can be derived from the work of Schwarz et al.(1991).For example, in one study reported by Schwarz et al.(1991), participants who were asked to generate six examples of how outgoing they were, judged themselves to be more outgoing than those who were asked to generate 12 examples. Participants in the 12-example condition apparently found it especially difficult to come up with 12 reasons for why they were outgoing, leading to the inference that they were not especially outgoing. If a similar process is responsible for the findings of Experiment 4, then participants should find it progressively more and more difficult to generate negative implications, leading to the 
inference that the negative event was not so bad after all.

Although feasible, this interpretation cannot account for the results. According to this difficulty interpretation, participants should have more difficulty in generating six negative implications than in generating either two or four negative implications. Therefore, participants in the six negative implications condition should infer that the negative event is not especially negative and the level of stress in this condition should be lower than in either of the other negative implications conditions. This was not the case. Although a difficulty explanation cannot account for the present findings, it is quite possible that perceivers' perceptions of difficulty would increase if they were asked to generate a very large number of implications or if they were asked to generate implications for an event that was not as negative as the ones generated in the present experiment. Therefore, although the difficulty interpretation does not account for the present results, it is a potentially viable way of reducing stress in other contexts. 6

TABLE 2: Mean Stress Ratings as a Function of the Number of Negative Implications

\begin{tabular}{lcc}
\hline Condition & $\mathrm{M}$ & $\mathrm{SD}$ \\
\hline $\begin{array}{l}\text { Highly negative experience } \\
\text { Highly negative experience and two } \\
\text { negative implications }\end{array}$ & 74.60 & 19.70 \\
$\begin{array}{l}\text { Highly negative experience and four } \\
\text { negative implications }\end{array}$ & 66.80 & 22.30 \\
$\begin{array}{l}\text { Highly negative experience and six } \\
\text { negative implications }\end{array}$ & 56.10 & 23.00 \\
\hline
\end{tabular}

\section{GENERAL DISCUSSION}

Experiments 1 through 4 provided evidence that perceivers' orientations to a stressing situation influence whether the addition of a mildly negative stressor decreased stress.In Experiment 1, an averaging effect was not obtained when perceivers concentrated on the implications of the entire situation and considered all of the consequences associated with the situation as a part of the whole.A veraging, however, was obtained when perceivers listed the negative implications of each stressor separately.This manipulation allowed participants to consider the situation in terms of the average amount of stress present rather than the total consequences 
of confronting one stressor on top of another.

In Experiment 2 and 3, thinking about a mildly negative event in addition to a highly negative one lowered stress levels below that produced by the highly negative event alone. However, this effect was attenuated by requiring perceivers to overtly appraise each stressor before reporting their overall stress reactions. In Experiment 4 , the stress surrounding a highly negative experience was reduced by having participants think about a few of the negative implications associated with a highly negative event.Because there are usually many implications associated with a highly negative experience, each event is a subset of the total experience, and each implication is usually less negative than the complete experience itself. Therefore, by separating the event in this way, adding thoughts about a small subset of the negative event to those associated with the entire negative experience, stress levels were reduced below that produced by the negative experience alone.7

Our research tact to date has been to demonstrate the counterintuitive findings obtainable when predicting from the AS model.In particular, there have been multiple demonstrations, in this and other work, that adding stressors or evaluative others to a context can result in decrements in experiential stress.In the present research, we add to this body of knowledge by demonstrating preconditions that increase the tendency for stress averaging to occur.In particular, this research demonstrated that stress averaging occurred when individuals oriented toward stressful events in a manner that resulted in a separation of the stimuli.For example, writing implications of each stressor separately was assumed to allow for the appraisal of each stressor in terms of its own properties rather than viewing it as a part of the undifferentiated definition of the total situation.Draw ing attention to the distinct properties of the stressor per se allows for an integration of the event in terms of its contribution to the average amount of stress present, which can have a diminutive influence.For example, while having to take the children to day care may be a somewhat stressing event, and may have an additive effect on parents' stress levels, when this event is considered separately from other stressors present in one's day (e.g., having to fire an employee), it may decrease rather than increase one's experiential stress. But, if one's orientation to the day's events takes the perspective of "one thing added to another," or a part of the undifferentiated definition of the total situation, it may have an incremental effect on experienced stress. 
The present research demonstrated that mere separation of stimuli per se is not sufficient to produce averaging effects. Even when the context induced the separation of stressors (e.g., via independent consideration of interpolated events), other factors, such as the overt expression of stress levels, may mitigate the beneficial influence of event separations.Furthermore, if the properties (e.g., implications) of the two events are not sufficiently discrepant, the addition of this stressor would not lower the combined average of the person's initial level of stress, a mild (carpooling event) and high stressor (e.g., firing an employee). For example, assume that the firing stressor is 3, carpooling is a 2.5, and the person's initial (baseline) level is .5. When carpooling is added, the average consists of the stimulus value of carpooling (2.5), the firing event (3), and the initial level (.5). The average then would be $2[(3+2.5+.5) / 3]$ and is higher than that associated with the firing event alone $[(3+.5) /$ $2=1.75]$. But if the firing event was appraised as 8 , then the carpooling event would have a diminutive effect on stress $[(8+2.5+.5) / 3=3.66]$, which is lower than the firing event's level alone $[(8+.5) / 2=4.25]$. Therefore, when a distinct stressor is above the person's initial baseline level but significantly lower than other stressors present in the context, the addition of this stressor is expected to lower stress. However, when the stressor is above baseline but is not appraised as significantly different from other stressors, its addition should not lower experiential stress and may indeed raise stress.

Thus, both stimulus property discrepancies and one's orientation to the context are factors that determine whether additional stressors will decrease or increase experiential stress. It should be pointed out that although this article demonstrates that the distinctiveness or separation of stressors resulted in stress reduction effects, distinctiveness also plays a role in obtaining summative effects as well. As discussed in J.J. Seta et al.(1989), audience members supply a distinct source of consequences and may raise the total consequences that accrue to the performer, for example, by increasing the probability of error detection or the number of tomatoes that are tossed. Similarly, negative events or stressors also supply a distinct source of potentially negative consequences, for example, by depleting the pool of available coping resources or increasing cortisol levels. Thus, different from contexts of "cold" impression formation, "hot" situations involving negative affect, stress, or more generally, negative consequences are contexts in which events are especially likely to contribute to the accumulation of consequences, thereby 
increasing anxiety or stress (see J.J.Seta et al., 1989, for a discussion of when summation occurs in "cold" contexts).

The AS model also leads to predictions that adding stressors to a context may lead to reductions in stress by means other than the application of averaging processes per se. One method by which this may be accomplished is via distraction. Each stimulus (e.g., stressor) within the model is influenced by a weighting factor, which is conceptualized as the importance or attentional focus associated with the stimulus. The addition of stimuli to a person's perceptual field may draw attentional focus away from high-level stressors. In this case, distraction would have the theoretical function of decreasing the weight associated with the high stressor, resulting in a smaller weighted sum. Of course, the stressing properties of the added stressor must be sufficiently low as to have only a marginal effect on the total sum of all stressors present in the context. For example, the night before one is scheduled to take a stressing exam, a student may spend time doing chores such as laundry. Although laundry chores may be somewhat stressing, this activity can be distracting and may decrease the salience of the upcoming exam. In such circumstances, the stress value associated with the chore may be low enough to compensate for the impact its addition has on the overall sum of stress psychologically present in the setting and the student may feel less stressed than if he was not engaged in this activity.

Given that the applicable domain of the model is a complex stimulus field (e.g., heterogeneous audience or other stressful settings), it is possible that assimilation processes also can influence the perceptual weight associated with the various stimuli within the AS model. Stim ulus assimilation may result in decrements in experienced stress under circumstances in which a relatively intense stressor is perceived as less threatening (i.e., weighted less) in the presence of a less intense stressor than when it exists in isolation. The resultant sum of the heterogeneous stressing context may be less than the stress value of the intense stressor per se when the weight of the intense stressor has been assimilated toward the value of a lower level stressor. For example, a highly evaluative expert observer may appear less threatening when he is in the context of less expert audience members than alone. In this case, the weight associated with the expert observer may decrease sufficiently to offset any additions of anxiety produced by adding less expert audience members. 
Our research thus far has concentrated on the role of averaging per se in reducing stress and anxiety. Although the effects of distraction and assimilation described above are theoretically derivable from the AS model, they are unlikely to have been responsible for our current or past findings. This is the case for several reasons. First, the potential role of distraction was minimized in this research by requiring participants to concentrate on high stressors through requirements to generate these stimuli from their life experiences and writing about their implications. These procedures make it unlikely that the high-stressing stimuli could be ignored. In addition, the lack of order effects provides further support that the addition of a low-level stressor did not cause participants to ignore the implications of the high-level stressor. Specifically, when the addition of a low-level stressor reduced stress, it did not matter if the stressor preceded or followed the high stressor, as substantiated by the lack of order main effects or interactions.

This would not be expected from a distraction account. That is, from such an account, one would have expected that the presence of the low-level stressor would have had more or less of an impact depending on its serial order vis-à-vis the high stressor. For example, the presence of a low-level stressor might be expected to decrease the weight of a high-level stressor if it followed this stressor but not if it preceded it.

Our research has employed stimuli that were very discrepant in their stress levels. This procedure should minimize the likelihood of stimulus assimilation because assimilation effects have been found to occur under conditions in which the discrepancies between stimuli are relatively small (e.g., Sherif \& Hovland, 1961). In the present research, as in our past work, we expect to find diminutive effects of adding low-level stressors under conditions in which the stimulus value of these stressors are very discrepant from that of the higher level stressors. As previously discussed, reductions in stress are especially likely when there are relatively large discrepancies between stimuli. For example, in past research, we found reductions in anxiety when high school students were added to an audience of faculty but not when undergraduates were added to an audience of graduate students (e.g., J. J. Seta et al., 1989).

The results of the interpolated conditions of Experiments 2 and 3 argue against an interpretation in which reductions in stress would be due to reductions in the negativity (scale value) of the highly negative stressor in 
the presence of a mildly negative stressor. For example, assume that the negativity associated with the highly negative event when experienced in isolation is 70 , while its value in the presence of a mildly negative event is reduced to 35 .Further, assume that the mildly negative event has a negativity value of 30 .In this situation, the sum of the two stressors $(35+30)$ would be less than the stress associated with the negative event in isolation (70). The results of the interpolated conditions of Experiments 2 and 3 are not supportive of the aforementioned possibility. In the interpolated conditions, participants were asked to report the level of stress that they experienced from thinking about each event. In these studies, the stress levels reported in the highly negative event alone conditions were sometimes slightly lower or higher than those reported in the mixed conditions, but were never significantly higher than those reported in the mixed conditions (e.g., 1 highly negative +1 mildly negative event, all ts less than .4, ps > .3). For example, in Experiment 3 participants reported a stress level of 89.4 when the highly negative event was presented in isolation. When it was presented along with a mildly negative event, participants reported an average stress level of 86.8 for the highly negative event and an average stress level of 48.4 for the mildly negative event. The stress level reported in the mixed condition was not significantly different from that reported in the highly negative event alone condition, $t=.22$, ns.

\section{Related Issues}

If averaging requires more cognitive capacity than does summation, then a person's level of cognitive capacity should influence the likelihood of averaging and a response from a cognitively overloaded person may not be especially likely to involve averaging. In a similar view, a situation in which the central tendency is difficult to find would preclude individuals who are not especially willing or capable from finding the central tendency that defines that situation, thereby reducing the effects of averaging. As discussed in our previous research (see J.J.Seta et al., 1989), the addition of a stressor or an audience member can influence both the averaging and summation component of the model. Pre dictions about the effect of this addition, therefore, depend on which component is most heavily influenced by this addition (e.g., under some circumstances, the addition of a stimulus can have a negligible effect on the summative component while having a major effect on 
the averaging component) (see J.J.Seta et al., 1989, for further discussion).

Our analysis and findings (e.g., C. E. Seta \& Seta, 1996; J.J.Seta et al., 1989, 1991) support and extend our work on averaging and summation as well as the work of Kahneman and associates (e.g., Kahneman, Fredrickson, Schreiber, \& Redelmeier, 1993; Redelmeier \&

Kahneman, 1996).Kahneman and associates proposed a weighted averaging model for perceptions of pain in that participants preferred a pain sequence that involved both a severe and moderate pain event over one that just involved a severely painful event (e.g., Kahneman et al., 1993). The results of this line of research are consistent with the averaging component of our analysis. There are differences, however, between the two approaches. Our averaging/summation analysis, for example, incorporates both averaging and summation, whereas their view concentrates exclusively on averaging. Consequently, we delineate situations in which adding a low-level stressor or pain sequence is likely to reduce as well as increase the impact of a stimulus configuration. Nevertheless, the combined results of both lines of work provide evidence that perceptions of both stress and pain can be reduced by the addition of one or a few relatively mild negative events to a relatively severe negative one.

\section{CONCLUSION}

These studies have both theoretical and applied implications. At the theoretical level, they support predictions that highlight the importance of understanding how individual integration strategies influence stress reactions. In so doing, they provide evidence for the importance of the psychological context in influencing the impact of central tendencies, such as averaging. At the applied level, our results provide the foundation for procedures that can reduce perceptions of stress following negative life experiences. 


\section{NOTES}

1.It may be the case that in many situations a person's initial level when entering a setting is not a primary factor in that it may not differ markedly from the individual's typical level. In such a case, the person's initial level might establish a neutral point or a basis for calibrating incoming stimuli. In the present series of studies, there is no reason to assume that initial levels would be different across conditions within a given study. In addition, there is no reason to presume that the relative weights assigned to stimuli did not remain relatively constant within each mixed stress condition.

2.Because both order main effects and the Order $\times$ Condition interaction were not significant, order was collapsed across conditions.

3.We examined the implications of approximately $50 \%$ of our participants $(N=114)$ to determine if the implications that they listed were negatively valenced. All of the listed implications were negative. This was also the case in all of the studies reported. Participants also filled out Rosenberg's Self-Esteem Scale (Rosenberg, 1965).Self-esteem was dropped from subsequent analyses because when this factor was entered into a 2 (high or low self-esteem) $\times 3$ (control, separate, total) ANOVA, the analysis did not reveal a main effect for self-esteem $(F<.1)$ or a Self-Esteem $\times$ Condition interaction $(F<.4)$. The cutoff score for low self-esteem was 33 and below. It should be pointed out that we did not expect self-esteem to influence the strength of averaging in this particular study. We included it so that these results may be compared to other studies in which this variable is expected to influence the strength of averaging.

4.The highly negative events that were reported occurred, on average, approximately 46 months before the experiment. The minor events occurred, on average, approximately 10 months before the experiment.

5.W e included order (highly negative event first or last) in a between-participant design. The design also included the two mixed stressor conditions and the single and multiple conditions. The ANOVA performed on these data indicated that order did not interact with either condition. Nevertheless, it is interesting that in the final response condition, the highly negative event and three mildly negative events condition was influenced more by order than in any of the other conditions. Participants' reports of stress were approximately 20 points higher when the highly negative event came last than when it came first. Per haps participants weigh the highly negative event especially heavily when it is proceeded by several mildly negative events.

6.Other alternative explanations could be formulated based on assumptions that implications generated earlier in the task were more negative than later implications. These explanations do not account 
for the present data; analyses of the rated negativity of each implication revealed no significant order effects (all Fs < 1.05).

7.In Experiments 1 through 4, individual reactions were tested shortly after stimulus exposure. A question arises as to whether different results would have been obtained if participants' responses were measured after a substantial amount of time had elapsed. Although we would expect that the effects of our manipulations would weaken over time, we have no reason to believe that the pattern of our results would have reversed if we delayed the testing of participants' responses.

\section{REFERENCES}

Hobfoll, S.E.(1988a). Conservation of resources: A new attempt at conceptualizing stress. American Psychologist, 44, 513-524.

Hobfoll, S.E.(1988b). The ecology of stress. Washington, DC: Hemisphere.

Kahneman, D., Fredrickson, B. L., Schreiber, C. A.,\&Redelmeier, D. A. (1993).When more pain is preferred to less: Adding a better end. Psychological Science, 4, 401-405.

Lazarus, R.S.(1966). Psychological stress and coping process. New York: McGraw-Hill.

Lazarus, R. S., Delongis, A., Folkman, S., \& Gruen, R. (1985). Stress and adaptation outcomes: The problem of confounded measures. American Psychologist, 40, 770-779.

Lazarus, R.S. , \& Folkman, S.(1984). Stress, appraisal, and coping. New York: Springer.

Linville, P.W ., \& Fischer, W.(1991).Preferences for separating or combing events. Journal of Personality and Social Psychology, 60, 5-23.

Lyubomirsky, S., \& Nolen-Hoeksema, S.(1995).Effects of self-focused rumination on negative thinking and interpersonal problem solving. Journal of Personality and Social Psychology, 69, 176-190.

Martin, L.L. , \& Tesser, A.(1996).Some ruminate thoughts. In R.S. Wyer Jr.(Ed. ), Ruminative thoughts (pp.1-47).Mahwah, NJ: Lawrence Erlbaum.

Random House college dictionary (1st ed.).(1984).New York: Random House.

Redelmeier, D.A. , \& Kahneman, D.(1996).Patients' memories of painful medical treatments: Real time and retrospective evaluations of two minimally invasive procedure. Pain, 66, 3-8. 
Rosenberg, M.(1965). Society and the adolescent self-image.Princeton, NJ: Princeton University Press.

Schwarz, N., Bless, H., Strack, F., Klump, G., Rittenauer-Schatker, H., \& Simons, A.(1991).Ease of retrieval as information. Another look at the availability heuristic. Journal of Personality and Social Psychology, 61, 195-202.

Schwarz, N., \& Clore, G.L.(1983).Mood, misattribution and judgments of well-being: Informative and directive functions of affective states. Journal of Personality and Social Psychology, 45, 513-523.

Seta, C.E. , \& Seta, J.J.(1992).Increments and decrements in mean arterial pressure as a function of audience composition: An averaging and summation analysis. Personality and Social Psychology Bulletin, $18,173-181$.

Seta, C.E. , \& Seta, J.J.(1996).When more is less: An averaging/ summation analysis of social anxiety. Journal of Research in Personality, 30, 496-509.

Seta, J.J. , Crisson, J.E. , Seta, C.E. , \&Wang, M.A.(1989).T ask performance and perceptions of anxiety: Averaging and summation in an evaluative setting. Journal of Personality and Social Psychology, 56, 387-396.

Seta, J.J. , Seta, C.E. , \&Wang, M.A.(1991).Feelings of negativity and stress: An averaging-summation analysis of impressions of negative life experiences. Personality and Social Psychology Bulletin, 17, 376-384.

Seta, J.J. , Wang, M.A. , Crisson, J.E. , \& Seta, C.E.(1989).Audience composition and felt anxiety: Impact averaging and summation. Basic and Applied Social Psychology, 10, 57-72.

Seyle, H.(1956). The stress of life. New York: McGraw-Hill. Sherif, M., \& Hovland, C.I.(1961). Social judgment: Assimilation and contrast effects in communication and attitude change. New Haven, CT: Yale University Press.

Strack, F., Blaney, P.H. , Ganellen, R.J. , \& Coyne, J.C.(1985). Pessi mistic self-preoccupation performance deficits and depression. Journal of Personality and Social Psychology, 49, 1076-1085. 